MITP/15-085 (Mainz)

August 3, 2021

\title{
Towards an assessment of the ATLAS data on the branching ratio $\Gamma\left(\Lambda_{b}^{0} \rightarrow \psi(2 S) \Lambda^{0}\right) / \Gamma\left(\Lambda_{b}^{0} \rightarrow J / \psi \Lambda^{0}\right)$
}

\author{
Thomas Gutsche, ${ }^{1}$ Mikhail A. Ivanov, ${ }^{2}$ Jürgen G. Körner, ${ }^{3}$ Valery E. Lyubovitskij,,${ }^{1,4,5}$ and Pietro Santorelli ${ }^{6,7}$ \\ ${ }^{1}$ Institut für Theoretische Physik, Universität Tübingen, Kepler Center for Astro and Particle Physics, \\ Auf der Morgenstelle 14, D-72076, Tübingen, Germany \\ ${ }^{2}$ Bogoliubov Laboratory of Theoretical Physics, Joint Institute for Nuclear Research, 141980 Dubna, Russia \\ ${ }^{3}$ PRISMA Cluster of Excellence, Institut für Physik, \\ Johannes Gutenberg-Universität, D-55099 Mainz, Germany \\ 4 Department of Physics, Tomsk State University, 634050 Tomsk, Russia \\ ${ }^{5}$ Mathematical Physics Department, Tomsk Polytechnic University, Lenin Avenue 30, 634050 Tomsk, Russia \\ ${ }^{6}$ Dipartimento di Fisica, Università di Napoli Federico II, \\ Complesso Universitario di Monte Sant' Angelo, Via Cintia, Edificio 6, 80126 Napoli, Italy \\ ${ }^{7}$ Istituto Nazionale di Fisica Nucleare, Sezione di Napoli, 80126 Napoli, Italy
}

\begin{abstract}
Recently the ATLAS Collaboration at CERN reported on the measurement of the branching ratio $R_{\Lambda_{b}}=\Gamma\left(\Lambda_{b}^{0} \rightarrow \psi(2 S) \Lambda^{0}\right) / \Gamma\left(\Lambda_{b}^{0} \rightarrow J / \psi \Lambda^{0}\right)$. The measured branching ratio $R_{\Lambda_{b}}=0.501 \pm 0.033$ (stat) \pm 0.019 (syst) was found to be lower than the covariant quark model prediction of $R_{\Lambda_{b}}=$ $0.8 \pm 0.1$ calculated by us recently. We present a detailed analysis of the branching ratio $R_{\Lambda_{b}}$ using a model-independent framework for the heavy-to-light form factors based on results from our previos papers.
\end{abstract}

PACS numbers: 12.39.Ki,13.30.Eg,14.20.Jn,14.20.Mr

Keywords: relativistic quark model, light and heavy baryons, decay rates

\section{INTRODUCTION}

Recently the ATLAS Collaboration at CERN reported on the measurement of the branching ratio $R_{\Lambda_{b}}=$ $\Gamma\left(\Lambda_{b}^{0} \rightarrow \psi(2 S) \Lambda^{0}\right) / \Gamma\left(\Lambda_{b}^{0} \rightarrow J / \psi \Lambda^{0}\right)$ [1]. The measured branching ratio $R_{\Lambda_{b}}=0.501 \pm 0.033$ (stat) \pm 0.019 (syst) was found to be lower than the expectation from the covariant quark model calculation 2-8] $R_{\Lambda_{b}}=0.8 \pm 0.1$ done by us recently [6]. Note, in Ref. [6] we have only listed the central value of $R_{\Lambda_{b}}=0.8 \pm 0.1$. The error on the branching ratio was determined using several overall fits to a wide spectrum of data on heavy hadron decays all with similar good $\chi^{2}$ values. The set of fit values lead to branching ratios within 0.1 deviation from 0.8 . We mention that there have been a number of theoretical quark model calculations for the decay $\Lambda_{b} \rightarrow \Lambda+J / \psi$ based on the factorization hypothesis [9]-[15] two of which we will return to when we present our numerical results.

In Ref. [6] we have presented a detailed analysis of the branching ratio $R_{\Lambda_{b}}$ using covariant quark model for the heavy-to-light form factors based on results from our previos papers. In particular, we have shown that our model transition form factors (see Ref. [7] for heavy-tolight transitions and Ref. [8] for heavy-to-heavy transitions) can be approximated to a high accuracy by the double-pole representation

$$
F\left(q^{2}\right)=\frac{F(0)}{1-a q^{2} / M_{1}^{2}+b q^{4} / M_{1}^{4}},
$$

where $M_{1}$ is the mass of the initial baryon and $a$ and $b$ are fit parameters. In the examples studied by us we noticed that the fit parameters are approximately related by $b \approx a^{2} / 4$, i.e. the $q^{2}$ behavior of our form factors is very close to a dipole form

$$
F\left(q^{2}\right)=\frac{F(0)}{\left(1-q^{2} / M_{d}^{2}\right)^{2}}
$$

where $M_{d}$ is the dipole mass. The scale set by this mass is close to the value of the $B_{s}$ meson mass $M_{B_{s}}=$ $5.367 \mathrm{GeV}$ in the case of the $b \rightarrow s$ transitions.

The main objective of the present paper is to show that the explicit value of the dipole mass is crucial to understand the branching ratio $R_{\Lambda_{b}}$. Our main result is that $M_{d} \sim M_{B_{s}}$ leads to $R_{\Lambda_{b}} \sim 1$, while increasing/decreasing values of $M_{d}$ lead to decreasing/increasing values of $R_{\Lambda_{b}}$. In particular, the central ATLAS value $R_{\Lambda_{b}} \sim 0.5$ would require a relatively large value of the dipole mass $M_{d} \sim 10 \mathrm{GeV}$.

We start with the definition of the transition amplitude of the process $B_{1}\left(p_{1}\right) \rightarrow B_{2}\left(p_{2}\right)+W_{\text {off-shell }}(q)$ which are described by the vector and axial vector current matrix elements $M_{\mu}^{V / A}\left(\lambda_{1}, \lambda_{2}\right)=\left\langle B_{2}, \lambda_{2}\left|J_{\mu}^{V / A}\right| B_{1}, \lambda_{1}\right\rangle$. The matrix elements can be expanded in terms of a complete set of invariants:

$$
\begin{aligned}
M_{\mu}^{V}\left(\lambda_{1}, \lambda_{2}\right) & =\bar{u}_{2}\left(p_{2}, \lambda_{2}\right)\left[F_{1}^{V}\left(q^{2}\right) \gamma_{\mu}-\frac{F_{2}^{V}\left(q^{2}\right)}{M_{1}} i \sigma_{\mu q}\right. \\
& \left.+\frac{F_{3}^{V}\left(q^{2}\right)}{M_{1}} q_{\mu}\right] u_{1}\left(p_{1}, \lambda_{1}\right)
\end{aligned}
$$


and

$$
\begin{aligned}
M_{\mu}^{A}\left(\lambda_{1}, \lambda_{2}\right) & =\bar{u}_{2}\left(p_{2}, \lambda_{2}\right)\left[F_{1}^{A}\left(q^{2}\right) \gamma_{\mu}-\frac{F_{2}^{A}\left(q^{2}\right)}{M_{1}} i \sigma_{\mu q}\right. \\
& \left.+\frac{F_{3}^{A}\left(q^{2}\right)}{M_{1}} q_{\mu}\right] \gamma_{5} u_{1}\left(p_{1}, \lambda_{1}\right)
\end{aligned}
$$

where $M_{1}$ and $M_{2}$ are the masses of the initial and final baryon, $\sigma_{\mu q}=\frac{i}{2}\left(\gamma_{\mu} \not q-\not q \gamma_{\mu}\right)$ and $q=p_{1}-p_{2}$. The labels $\lambda_{i}= \pm \frac{1}{2}$ denote the helicities of the two baryons. For completeness we have also included the form factors $F_{3}^{V / A}$ even though they do not contribute to the process $\Lambda_{b} \rightarrow \Lambda+J / \psi, \psi(2 S)$. They would determine the rate for the decay $\Lambda_{b} \rightarrow \Lambda+\eta_{c}$.

In the heavy quark limit (HQL) the matrix element for the heavy-to-light $b \rightarrow s$ transition is given in terms of two $\left(f_{1}, f_{2}\right)$ form factors [16 18]. The HQL form factors depend on the variable $p_{2} \cdot v_{1}$, where $v_{1}=p_{1} / M_{1}$ is the four-velocity of the $\Lambda_{b}$. The matrix element now reads

$$
M_{\mu}^{V-A}=\bar{u}\left(p_{2}\right)\left(f_{1}\left(p_{2} v\right)+\not f_{2}\left(p_{2} v\right)\right) O_{\mu} u(v)
$$

where $O_{\mu}=\gamma_{\mu}\left(1-\gamma^{5}\right)$. In the HQL we have used the heavy ( $b$ quark) mass expansion for the $\Lambda_{b}$ mass [ $[$ ]

$$
M_{\Lambda_{b}}=m_{b}+\bar{\Lambda}+\mathcal{O}\left(1 / m_{b}\right)
$$

and keep the two leading terms in the expansion - the heavy quark mass $m_{b}$ and the so-called binding energy $\bar{\Lambda}=\mathcal{O}\left(m_{b}^{0}\right)$. The value $\bar{\Lambda}=0.53 \mathrm{GeV}$ is fixed using experimental values for $M_{\Lambda_{b}}=5.6194 \mathrm{GeV}$ [19] and a model value for the constituent mass of the $b$ quark $m_{b}=$ $5.09 \mathrm{GeV}$. The constituent mass of the $b$ quark was fixed from an analysis of a wide spectrum of data on heavy hadron decays in our approach.

In the HQL the six form factors $F_{1,2,3}^{V / A, \mathrm{HQL}}$ become related to the HQL form factors $f_{1,2}$ as follows [ $\left.\begin{array}{lll}16 & 18\end{array}\right]$

$$
\begin{aligned}
& F_{1}^{V, \mathrm{HQL}}=F_{1}^{A, \mathrm{HQL}}=f_{1}+\frac{M_{2}}{M_{1}} f_{2}, \\
& F_{2}^{V, \mathrm{HQL}}=F_{2}^{A, \mathrm{HQL}}=-F_{3}^{V, \mathrm{HQL}}=-F_{3}^{A, \mathrm{HQL}}=-f_{2}
\end{aligned}
$$

It is convenient to analyze the decay $\Lambda_{b} \rightarrow \Lambda+V$ in terms of the helicity amplitudes $H_{\lambda_{2} \lambda_{V}}^{V / A}$ which are linearly related to the invariant form factors $F_{i}^{V / A}$ (see details in Refs. [5] [8])

$$
H_{\lambda_{2} \lambda_{V}}^{V / A}=M_{\mu}^{V / A}\left(\lambda_{2}\right) \epsilon^{\dagger \mu}\left(\lambda_{V}\right),
$$

where $\lambda_{V}$ is the helicity of the vector meson. From angular momentum conservation, one has $\lambda_{1}=-\lambda_{2}+\lambda_{W}$.

The helicity amplitudes read (see e.g. Refs. [5 8 ])

$$
\begin{aligned}
H_{+\frac{1}{2}+1}^{V / A} & =\sqrt{2 Q_{\mp}}\left(F_{1}^{V / A} \pm \frac{M_{ \pm}}{M_{1}} F_{2}^{V / A}\right), \\
H_{+\frac{1}{2} 0}^{V / A} & =\frac{\sqrt{Q_{\mp}}}{\sqrt{q^{2}}}\left(M_{ \pm} F_{1}^{V / A} \pm \frac{q^{2}}{M_{1}} F_{2}^{V / A}\right),
\end{aligned}
$$

where we make use of the abbreviations $M_{ \pm}=M_{1} \pm M_{2}$ and $Q_{ \pm}=M_{ \pm}^{2}-q^{2}$. From parity or from an explicit calculation, one has $H_{-\lambda_{2},-\lambda_{V}}^{V / A}= \pm H_{\lambda_{2}, \lambda_{V}}^{V / A}$. The total left-chiral helicity amplitude is defined by the composition

$$
H_{\lambda_{2}, \lambda_{V}}=H_{\lambda_{2}, \lambda_{V}}^{V}-H_{\lambda_{2}, \lambda_{V}}^{A} .
$$

The weak nonleptonic decays $\Lambda_{b} \rightarrow \Lambda+J / \psi$ and $\Lambda_{b} \rightarrow \Lambda+\psi(2 S)$ are described by bilinear forms of the helicity amplitudes termed helicity structure functions. The relevant bilinear forms for the rate are $\mathcal{H}_{U}=\left|H_{+\frac{1}{2}+1}\right|^{2}+\left|H_{-\frac{1}{2}-1}\right|^{2}$ (unpolarized transverse) and $\mathcal{H}_{L}=\left|H_{+\frac{1}{2} 0}\right|^{2}+\left|H_{-\frac{1}{2} 0}\right|^{2}$ (longitudinal) where the rate is proportional to $\mathcal{H}_{U}+\mathcal{H}_{L}:=\mathcal{H}_{U+L}$. In the HQL the two helicity structure functions can be expressed in terms of the functions $f_{1}$ and $f_{2}$ as

$$
\begin{aligned}
\mathcal{H}_{U} & =4\left[\left(Q_{+}+Q_{-}\right)\left(f_{1}^{2}+f_{2}^{2}\right)+8 M_{1} M_{2} f_{1} f_{2}\right], \\
\mathcal{H}_{L} & =\mathcal{H}_{S}=\frac{2}{q^{2}}\left[Q_{+}\left(M_{-}\left[f_{1}+\frac{M_{2}}{M_{1}} f_{2}\right]+\frac{q^{2}}{M_{1}} f_{2}\right)^{2}\right. \\
& \left.+Q_{-}\left(M_{+}\left[f_{1}+\frac{M_{2}}{M_{1}} f_{2}\right]-\frac{q^{2}}{M_{1}} f_{2}\right)^{2}\right]
\end{aligned}
$$

The $\Lambda_{b} \rightarrow \Lambda+V$ decay rate is given by

$$
\begin{aligned}
\Gamma\left(\Lambda_{b} \rightarrow \Lambda+V\right) & =\frac{G_{F}^{2}}{32 \pi} \frac{\left|\mathbf{p}_{V}\right|}{M_{1}^{2}}\left|V_{c b} V_{c s}^{*}\right|^{2} C_{\mathrm{eff}}^{2} f_{V}^{2} M_{V}^{2} \\
& \times \mathcal{H}_{U+L} .
\end{aligned}
$$

where $\left|\mathbf{p}_{V}\right|=\sqrt{Q_{+} Q_{-}} /\left(2 M_{1}\right)$ is the three-momentum of the decay products in the rest frame of the parent baryon, $C_{\text {eff }}=-0.262$ is a combination of the relevant Wilson coefficients, $f_{V}$ is the decay constant $\left(f_{J / \psi}=415 \mathrm{MeV}\right.$, $\left.f_{\psi(2 S)}=295.6 \mathrm{MeV}\right)$ of the respective vector meson.

The branching ratio $R_{\Lambda_{b}}$ can be written in terms of a model independent factor $R_{M}$ given by

$$
R_{M}=\frac{\left|\mathbf{p}_{\psi(2 S)}\right|}{\left|\mathbf{p}_{J / \psi}\right|}\left(\frac{M_{\psi(2 S)} f_{\psi(2 S)}}{M_{J / \psi} f_{J / \psi}}\right)^{2}=0.535025
$$

and a model dependent factor $R_{\mathcal{H}}$ given by the ratio of the helicity structure functions $\mathcal{H}_{U+L}$ evaluated at $q^{2}=$ $M_{\psi(2 S)}^{2}$ and $q^{2}=M_{J / \psi}^{2}$

$$
R_{\mathcal{H}}=\left(\mathcal{H}_{U+L}\right)_{\psi(2 S)} /\left(\mathcal{H}_{U+L}\right)_{J / \psi}
$$

such that

$$
R_{\Lambda_{b}}=R_{M} \cdot R_{\mathcal{H}}
$$

Note that the branching ratio $R_{\Lambda_{b}}$ does not depend on the flavor and color dependent product of coefficients $V_{c b} V_{c s} C_{\text {eff. }}$.

Next we need to analyze the $q^{2}$-dependence of the ratio $R_{\mathcal{H}}$. According to the ATLAS data $R_{\mathcal{H}}$ must be close to 1 which implies a weak dependence of the helicity structure function on $q^{2}$ in the range between $q^{2}=M_{J / \psi}^{2} \simeq$ 
$9.591 \mathrm{GeV}^{2}$ and $q^{2}=M_{\psi(2 S)}^{2} \simeq 13.587 \mathrm{GeV}^{2}$. Our recent analysis showed that due the dipole-like behavior of the form factors (2) with $M_{d} \simeq M_{B_{s}}$ there is a sizeable growth of the rate structure function $\mathcal{H}_{U+L}$ from $q^{2}=M_{J / \psi}^{2}$ to $q^{2}=M_{\psi(2 S)}^{2}$. Quantitatively, the rapid growth of $\mathcal{H}_{U+L}$ between $q^{2}=M_{J / \psi}^{2}$ to $q^{2}=M_{\psi(2 S)}^{2}$ follows from the details of our dynamical quark model ansatz. Qualitatively, the growth of $\mathcal{H}_{U+L}$ results from the simple picture of a dipole behavior of the form factors characterized by the mass scale $\Lambda \simeq M_{B_{s}}$. The scale corresponds to the flavor composition of a $t$-channel meson exchange. Below we show details of our numerical analysis. In particular, we expose the dependence of $R_{\mathcal{H}}$ on the dipole mass $M_{d}$ and show that the ATLAS result can be reproduced only with a relatively large value of the dipole mass $M_{d} \sim 10 \mathrm{GeV}$ which exceeds the mass scale set by the $B_{s}$ meson mass by $86 \%$.

We want to emphasize that the dipole approximation is also a very good approximation for the $q^{2}-$ dependence of the $b \rightarrow c$ transition form factors in the decay $\Lambda_{b} \rightarrow \Lambda_{c} \ell^{-} \bar{\nu}_{\ell}[8]$. Again the dipole mass of $M_{d}=6.46-6.58 \mathrm{GeV}$ is close to the $(b \bar{c})$ mass scale of $6.28 \mathrm{GeV}$ set by the $B_{c}$ meson mass.

The details of the calculations of the covariant form factors for the $\Lambda_{b} \rightarrow \Lambda$ transition can be found in Ref. [6]. In particular, we used the following set of the constituent quark masses $m_{b}=5.09 \mathrm{GeV}, m_{s}=0.424 \mathrm{GeV}$, $m_{u}=m_{d}=0.235 \mathrm{GeV}$ and the actual value of $f_{\psi(2 S)}=$ $286.7 \mathrm{MeV}$.

In Figs. 1-6 we present plots of the $q^{2}$-dependence of our form factors from $q^{2}=0$ to $q_{\max }^{2}=\left(M_{\Lambda_{b}}-M_{\Lambda}\right)^{2}$. The two sets of Figs. 1-3 and 4-6 corresponds to the form factors $F_{1}^{V}$ and $F_{2}^{V}$, respectively. In particular, in Figs. 1 and 4 we compare the form factors in the exact case and in HQL. The leading $F_{1}^{V}$ form factors in the exact and in the HQL case can be seen to be very close to each other. In Figs. 2 and 5 we present a comparison of the exact form factors (dotted line), and the double-pole (curve 1) and dipole approximations (curve 2) to the form factors. An analogous comparison in the HQL is presented in Figs. 3 and 6 . One can see that the double-pole approximation lies practically on top of the exact form factors. Also the dipole approximation can be seen to be quite a reasonable approximation to both. The parameters for the double-pole and dipole approximations are summarized in Tables 1-4. We do not list the corresponding values for the form factor $F_{2}^{A}$ since $F_{2}^{A}$ is suppressed.

In Table 5 we present our results for the $\Lambda_{b} \rightarrow \Lambda+V$ branching ratios and the ratio $R_{\Lambda_{b}}$ considering different limiting cases: 1) exact, 2) exact taking into account only the leading form factors $F_{1}^{V}$ and $\left.F_{1}^{A}, 3\right) \mathrm{HQL}$ and 4) HQL taking into account only the leading form factors $F_{1}^{V, \mathrm{HQL}}=F_{1}^{A, \mathrm{HQL}}$. One can see that the restriction to the leading form factors $F_{1}^{V}$ and $F_{1}^{A}$ gives a qualitatively reasonable approximation for the evaluation of the branching ratios $B\left(\Lambda_{b} \rightarrow \Lambda+V\right)$.

In Fig. 7 we plot the dependence of the ratios $R_{\mathcal{H}}$ and
$R_{\Lambda_{b}}$ on the dipole mass using an approximation where $F_{2}^{V / A}=0$ and where the dominating form factors $F_{1}^{V}$ and $F_{1}^{A}$ have a dipole form. The plot encapsules the essence of the results found in Ref. [6] : for a dipole mass of $M_{d} \sim M_{B_{s}}$, which reflects the typical scale for the $b \rightarrow s$ baryon transitions, one finds $R_{\mathcal{H}} \sim 2$. Such a large value of $R_{\mathcal{H}}$ leads to a branching ratio $R_{\Lambda_{b}} \sim 1$ which exceeds the measured central value of $R_{\Lambda_{b}} \sim 0.5$ [1]. The ATLAS result can only be reproduced with $M_{d} \sim 10 \mathrm{GeV}$ which, as remarked on before, is far away from the mass scale set by the $B_{s}$ meson mass.

It is interesting to compare our results with the predictions of Refs. [14, 15] who have conveniently supplied parametrizations of their model form factors. The predictions of both models [14, 15] for the branching fraction $B\left(\Lambda_{b} \rightarrow \Lambda+J / \psi\right)$ are close to our result. However, the predictions for the branching fraction $B\left(\Lambda_{b} \rightarrow \Lambda+\psi(2 S)\right)$ considerably differ from our result leading to very different results on the branching ratio $R_{\Lambda_{b}}$. The authors of [14] obtain $R_{\Lambda_{b}}=0.65$, while the authors of [15] obtain a value of $R_{\Lambda_{b}}$ exceeding 1 . The result of Ref. [14] means that the value of their effective dipole mass is much larger than predicted by our approach.

For completeness we also present predictions of our model for analogous mesonic decays $B \rightarrow K+J / \psi, \psi(2 S)$ using the covariant quark model results given in [20]. The $B \rightarrow K+J / \psi, \psi(2 S)$ decay widths are calculated according to the formula

$$
\begin{aligned}
\Gamma(B \rightarrow K+V) & =\frac{G_{F}^{2}}{16 \pi} \frac{\left|\mathbf{p}_{V}\right|}{M_{1}^{2}}\left|V_{c b} V_{c s}^{*}\right|^{2} C_{\text {eff }}^{2} f_{V}^{2} M_{V}^{2}\left|H_{0}\right|^{2} \\
& =\frac{G_{F}^{2}}{4 \pi}\left|\mathbf{p}_{V}\right|^{3}\left|V_{c b} V_{c s}^{*}\right|^{2} C_{\text {eff }}^{2} f_{V}^{2} f_{+}^{2}\left(M_{V}^{2}\right)
\end{aligned}
$$

where $H_{0}$ is the scalar helicity amplitude given by 20]

$$
H_{0}=\frac{2 M_{1}\left|\mathbf{p}_{V}\right|}{M_{V}} f_{+}\left(M_{V}^{2}\right) .
$$

The form factor $f_{+}\left(M_{V}^{2}\right)$ multiplies the Lorentz-structure $\left(p_{1}+p_{2}\right)^{\mu}$ and has to be evaluated for $q^{2}=M_{V}^{2}$. The $B \rightarrow K$ transition form factors calculated in [20] are very close to a monopole formula

$$
f_{+}\left(q^{2}\right)=\frac{f_{+}(0)}{1-q^{2} / M_{m}^{2}}
$$

where $M_{m}$ is the monopole mass. In the case of the mesonic $b \rightarrow s$ transitions the monopole mass $M_{m}$ is of the order of $5 \mathrm{GeV}$. As before the branching ratio $R_{B}=\Gamma(B \rightarrow \psi(2 S) K) / \Gamma(B \rightarrow J / \psi K)$ can be written in terms of the two factors $R_{B}=R_{M} R_{\mathcal{H}}$, where $R_{M}=0.515792$ and $R_{\mathcal{H}}$ depends of the transtion form factor $f_{+}$according to Eq. (17). In Fig. 8 we display the dependence of the ratios $R_{\mathcal{H}}$ and $R_{B}$ on the value of the monopole mass $M_{m}$. Due to the monopole form of the mesonic transition form factors the dependence of $R_{\mathcal{H}}$ and thereby $R_{B}$ on the monopole mass is weaker than in the baryon case. In particular, $R_{B}$ changes from 0.39 to 
0.24 when $M_{m}$ changes from 5 to $10 \mathrm{GeV}$. The exact value $R_{B}$ predicted by our approach is $R_{B}=0.34$. This value is smaller than the experimental data on $R_{B}: 0.611 \pm 0.019$ (fit) and $0.603 \pm 0.021$ (average) [19]. The smaller ratio predicted by our model is due to the smaller branching for the $B \rightarrow \psi(2 S) K$ mode $\operatorname{Br}(B \rightarrow \psi(2 S) K)=3.5 \times 10^{-4}$, while the experimental value is $(6.27 \pm 0.24) \times 10^{-4}$ (fit) and $(6.5 \pm 0.4) \times 10^{-4}$ (average) [19]. On the other hand, one should mention that our prediction for $\operatorname{Br}(B \rightarrow$ $\psi(2 S) K)$ is close to the results of the BaBar Collaboration $(4.9 \pm 1.6 \pm 0.4) \times 10^{-4}[21]$. In case of the $B \rightarrow J / \psi K$ mode we calculate $\operatorname{Br}(B \rightarrow J / \psi K)=9.6 \times 10^{-4}$, which is in good agreement with data $(10.27 \pm 0.31) \times 10^{-4}$ (fit) and $(10.24 \pm 0.35) \times 10^{-4}$ (average) [19].

The cases of nonleptonic meson and baryon $b \rightarrow s$ transitions appear to differ only by the power-scaling of the transition form factors. The different powers in the form factors (monopole in the meson sector and dipole in the baryon sector) leads to smaller values of the branching ratio in the meson sector, i.e. one has $R_{B}<R_{\Lambda_{b}}$. We hope that future experiments will clear up the picture on the branching ratios $R_{\Lambda_{b}}$ and $R_{B}$.

Let us summarize the main results of our paper. Using the covariant quark model we have analyzed the branching ratio $R_{\Lambda_{b}}=\Gamma\left(\Lambda_{b}^{0} \rightarrow \psi(2 S) \Lambda^{0}\right) / \Gamma\left(\Lambda_{b}^{0} \rightarrow J / \psi \Lambda^{0}\right)$ which was recently measured by the ATLAS Collaboration CERN [1]: $R_{\Lambda_{b}}=0.501 \pm 0.033$ (stat) \pm 0.019 (syst). The measurement disagrees with our prediction $R_{\Lambda_{b}}=$ $0.8 \pm 0.1$ calculated by us in Ref. [6] by 2.8 standard deviations. The error bars include variation of the quark masses $m_{b}=5.068 \pm 0.022 \mathrm{GeV}, m_{s}=0.426 \pm 0.002 \mathrm{GeV}$ and $m_{u}=m_{d}=0.238 \pm 0.003 \mathrm{GeV}$ and decay constant $f_{\psi(2 S)}$ from $286.7 \mathrm{MeV}$ (old value) to $295.6 \mathrm{MeV}$ (updated value). The same variation of the parameters and $f_{\psi(2 S)}$ in the meson case gives $R_{B}=0.35 \pm 0.05$. In the present paper we have presented arguments supporting our considerations on the dipole-like behavior of the leading $\Lambda_{b} \rightarrow \Lambda$ transition form factors characterized by a dipole mass $M_{d}$ close to the mass of the $B_{s}$ meson. Such dipole-like behavior is universal not only for heavyto-light, but also for heavy-to-heavy transitions. In the two cases the values of the dipole masses are found to be very close to the masses of the $\left(q_{1} \bar{q}_{2}\right)$ mesons that are active in the $q_{1} \rightarrow q_{2}$ current induced transition. In particular, the values of the dipole mass $M_{d}$ found for the $b \rightarrow c$ and $b \rightarrow s$ transitions are close to the $B_{c}$ and $B_{s}$ meson masses, respectively. It holds in both limiting cases - for finite values of the heavy quark masses and in the HQL.

\section{Acknowledgments}

This work was supported by the Tomsk State University Competitiveness Improvement Program and the Russian Federation program "Nauka" (Contract No. $0.1526 .2015,3854)$. M.A.I. acknowledges the support of the Mainz Institute for Theoretical Physics (MITP).
TABLE I: Parameters for the double-pole approximation for our form factors $F_{1}^{V}, F_{2}^{V}$ and $F_{1}^{A}$.

\begin{tabular}{crrr}
\hline & $F_{1}^{V}$ & $F_{2}^{V}$ & $F_{1}^{A}$ \\
\hline$f(0)$ & 0.107 & 0.043 & 0.104 \\
$a$ & 2.271 & 2.411 & 2.232 \\
$b$ & 1.367 & 1.531 & 1.328 \\
\hline
\end{tabular}

TABLE II: Parameters for the dipole approximation for our form factors $F_{1}^{V}, F_{2}^{V}$ and $F_{1}^{A}$.

\begin{tabular}{crrr}
\hline & $F_{1}^{V}$ & $F_{2}^{V}$ & $F_{1}^{A}$ \\
\hline$f(0)$ & 0.107 & 0.043 & 0.104 \\
$M_{d}(\mathrm{GeV})$ & 5.445 & 5.286 & 5.484 \\
\hline
\end{tabular}

TABLE III: Parameters for the double-pole approximation for our form factors $F_{1}^{V, \mathrm{HQL}}$ and $F_{2}^{V, \mathrm{HQL}}$ in HQL.

\begin{tabular}{ccc}
\hline & $F_{1}^{V, \mathrm{HQL}}$ & $F_{2}^{V, \mathrm{HQL}}$ \\
\hline$F(0)$ & 0.124 & 0.023 \\
$a$ & 2.259 & 2.464 \\
$b$ & 1.360 & 1.597 \\
\hline
\end{tabular}

TABLE IV: Parameters for the dipole approximation for our form factors $F_{1}^{V, \mathrm{HQL}}$ and $F_{2}^{V, \mathrm{HQL}}$ in HQL.

\begin{tabular}{cll}
\hline & $F_{1}^{V, \mathrm{HQL}}$ & $F_{2}^{V, \mathrm{HQL}}$ \\
\hline$F(0)$ & 0.107 & 0.043 \\
$M_{d}(\mathrm{GeV})$ & 5.458 & 5.240 \\
\hline
\end{tabular}

TABLE V: Branching ratios $B\left(\Lambda_{b} \rightarrow \Lambda+V\right)$ (in units of $10^{-4}$ ) and ratio $R_{\Lambda_{b}}$

\begin{tabular}{|c|c|c|c|c|}
\hline Quantity & Exact & $\begin{array}{c}\text { Exact } \\
F_{2}^{V / A}=0\end{array}$ & HQL & $\begin{array}{c}\text { HQL } \\
F_{2}^{V / A, \mathrm{HQL}}=0\end{array}$ \\
\hline$B\left(\Lambda_{b} \rightarrow \Lambda+J / \psi\right)$ & 8.90 & 7.34 & 9.55 & 10.27 \\
$B\left(\Lambda_{b} \rightarrow \Lambda+\psi(2 S)\right)$ & 7.25 & 5.96 & 7.40 & 8.52 \\
$R_{\Lambda_{b}}$ & 0.81 & 0.81 & 0.77 & 0.83 \\
\hline
\end{tabular}

TABLE VI: Comparison of our results for branching ratios $B\left(\Lambda_{b} \rightarrow \Lambda+V\right)$ (in units of $10^{-4}$ ) and ratio $R_{\Lambda_{b}}$ with approaches [14, 15] using their parametrization for form factors.

\begin{tabular}{|c|c|c|c|}
\hline Quantity & Our & Ref. [14] & Ref. [15] \\
\hline$B\left(\Lambda_{b} \rightarrow \Lambda+J / \psi\right)$ & 8.90 & 8.44 & 8.21 \\
$B\left(\Lambda_{b} \rightarrow \Lambda+\psi\right)$ & 7.25 & 5.48 & 9.35 \\
$R_{\Lambda_{b}}$ & 0.81 & 0.65 & 1.14 \\
\hline
\end{tabular}

M.A.I. and J.G.K. thank the Heisenberg-Landau Grant for support. 


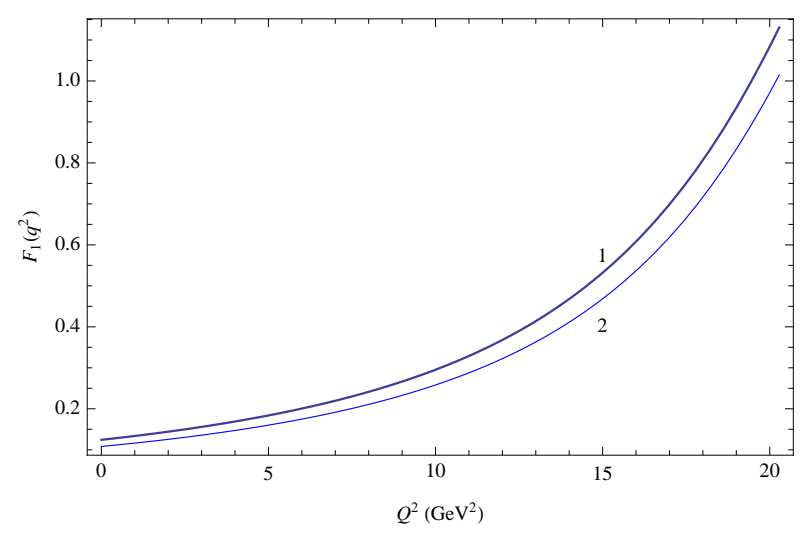

FIG. 1: Form factors $F_{1}^{V, \mathrm{HQL}}$ (curve 1) and $F_{1}$ (curve 2).

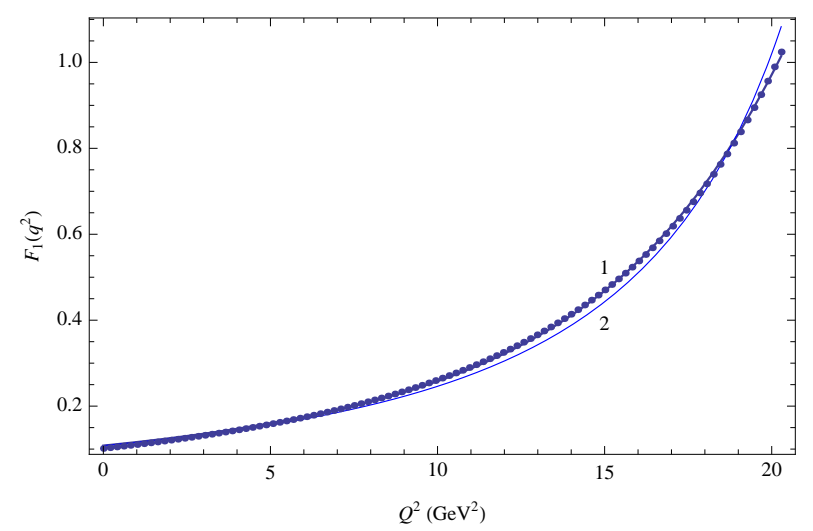

FIG. 2: Form factor $F_{1}^{V}$ : exact result (dotted line), double-pole approximation (curve 1) and dipole approximation (curve 2).

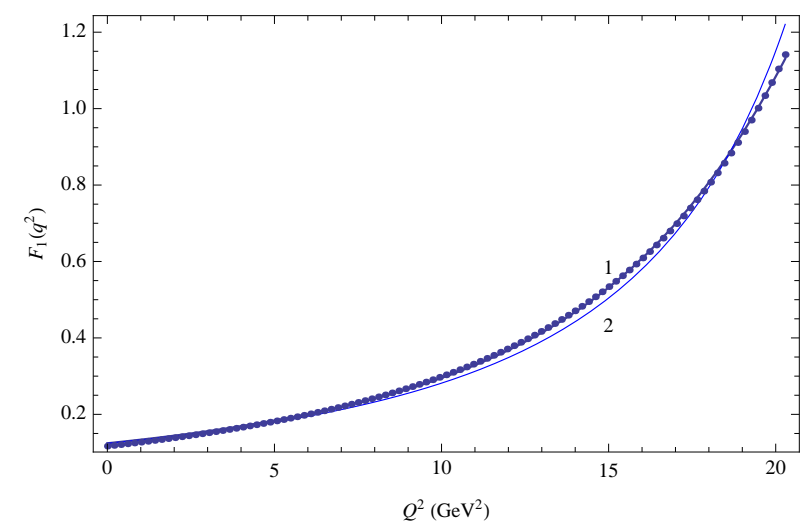

FIG. 3: Form factor $F_{1}^{V, \mathrm{HQL}}$ : exact result (dotted line), double-pole approximation (curve 1) and dipole approximation (curve 2).

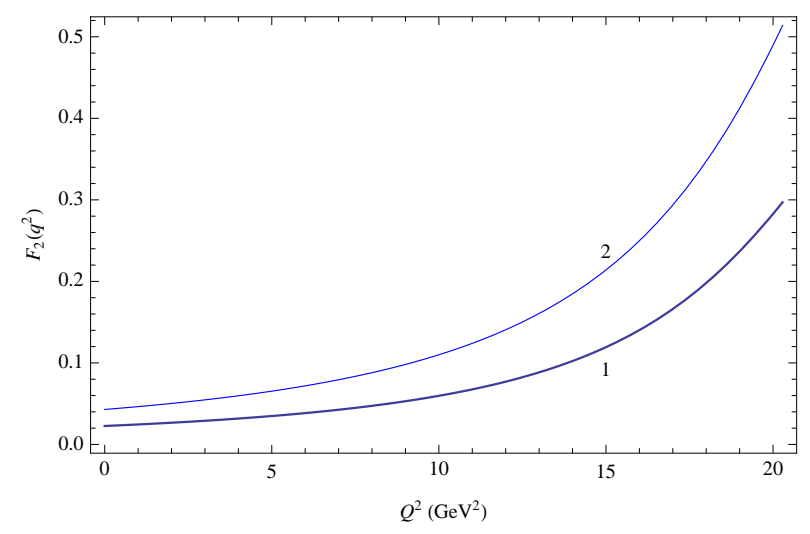

FIG. 4: Form factors $F_{2}^{V, \mathrm{HQL}}$ (curve 1) and $F_{2}$ (curve 2).

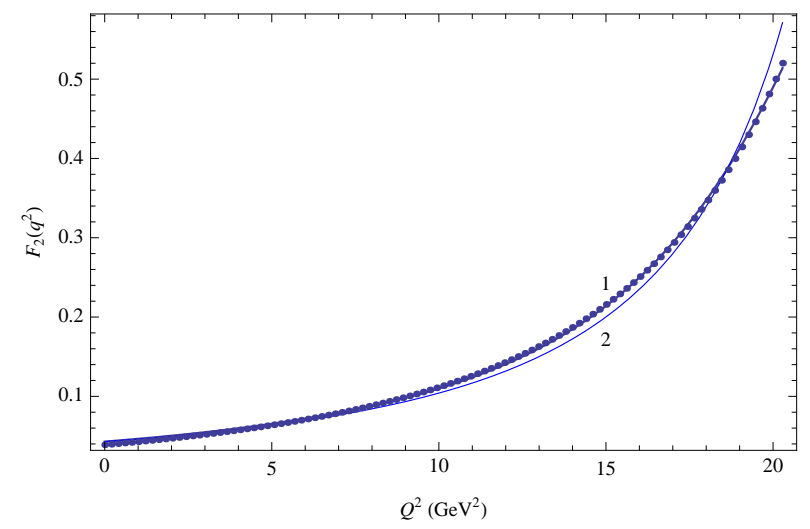

FIG. 5: Form factor $F_{2}^{V}$ : exact result (dotted line), double-pole approximation (curve 1) and dipole approximation (curve 2).

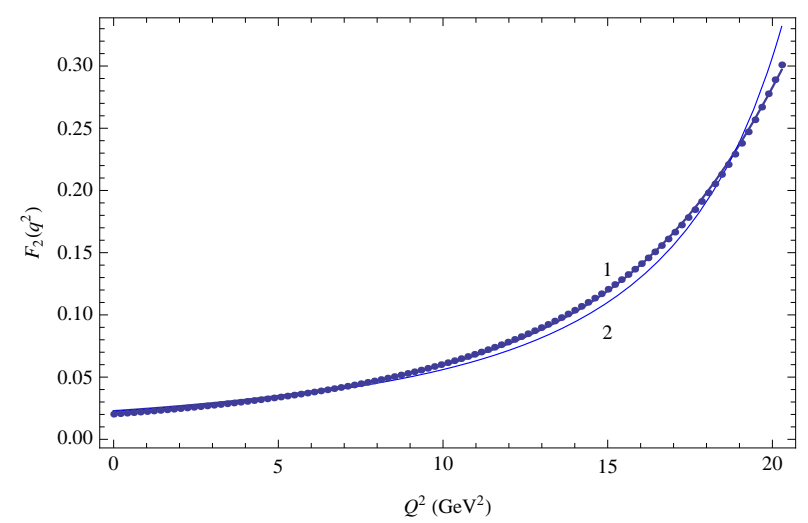

FIG. 6: Form factor $F_{2}^{V, \mathrm{HQL}}$ : exact result (dotted line), double-pole approximation (curve 1) and dipole approximation (curve 2). 


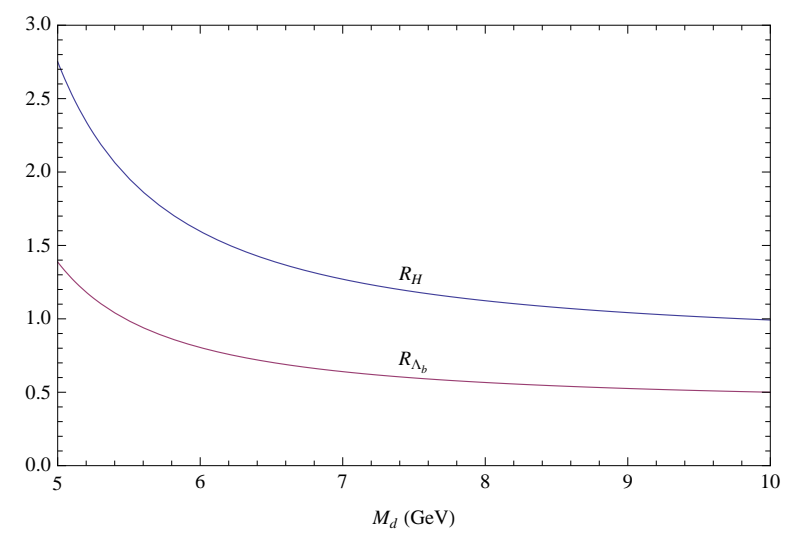

FIG. 7: Dependence of the ratios $R_{\mathcal{H}}$ and $R_{\Lambda_{b}}$ on the dipole mass $M_{d}$ for the nonleptonic $\Lambda_{b} \rightarrow \Lambda+J / \psi, \psi(2 S)$ transitions.

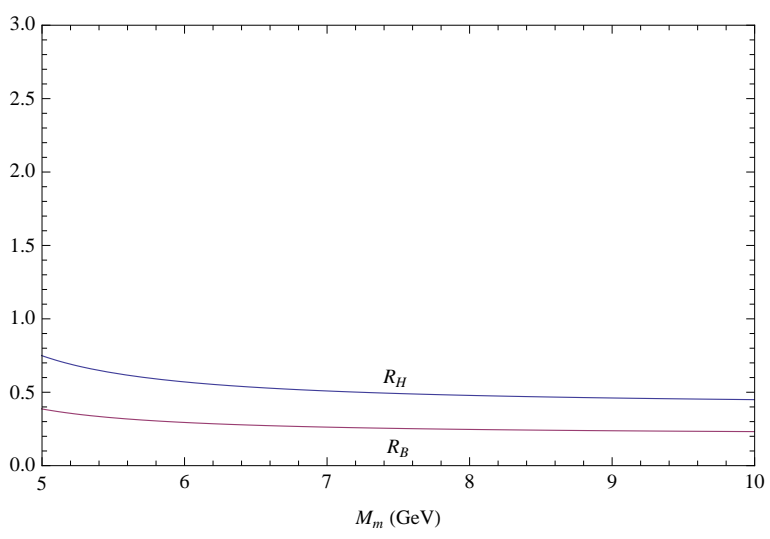

FIG. 8: Dependence of the ratios $R_{\mathcal{H}}$ and $R_{B}$ on the monopole mass $M_{m}$ for the nonleptonic $B \rightarrow K+J / \psi, \psi(2 S)$ transitions.
[1] G. Aad et al. (ATLAS Collaboration), arXiv:1507.08202 [hep-ex].

[2] M. A. Ivanov, M. P. Locher and V. E. Lyubovitskij, Few Body Syst. 21, 131 (1996) hep-ph/9602372.

[3] M. A. Ivanov, V. E. Lyubovitskij, J. G. Körner and P. Kroll, Phys. Rev. D 56, 348 (1997) arXiv:hep-ph/9612463.

[4] M. A. Ivanov, J. G. Körner, V. E. Lyubovitskij and A. G. Rusetsky, Phys. Rev. D 60, 094002 (1999) arXiv:hep-ph/9904421]; M. A. Ivanov, J. G. Körner, V. E. Lyubovitskij, M. A. Pisarev and A. G. Rusetsky, Phys. Rev. D 61, 114010 (2000) arXiv:hep-ph/9911425; A. Faessler, T. Gutsche, M. A. Ivanov, J. G. Körner, V. E. Lyubovitskij, D. Nicmorus and K. Pumsa-ard, Phys. Rev. D 73, 094013 (2006) arXiv:hep-ph/0602193; A. Faessler, T. Gutsche, B. R. Holstein, V. E. Lyubovitskij, D. Nicmorus and K. Pumsa-ard, Phys. Rev. D 74, 074010 (2006) arXiv:hep-ph/0608015; A. Faessler, T. Gutsche, B. R. Holstein, M. A. Ivanov, J. G. Körner and V. E. Lyubovitskij, Phys. Rev. D 78, 094005 (2008) arXiv:0809.4159 [hep-ph]]; A. Faessler, T. Gutsche, M. A. Ivanov, J. G. Körner and V. E. Lyubovitskij, Phys. Rev. D 80, 034025 (2009) arXiv:0907.0563 [hepph]]; T. Branz, A. Faessler, T. Gutsche, M. A. Ivanov, J. G. Körner, V. E. Lyubovitskij and B. Oexl, Phys. Rev. D 81, 114036 (2010) arXiv:1005.1850 [hep-ph]]; T. Gutsche, M. A. Ivanov, J. G. Körner, V. E. Lyubovitskij and P. Santorelli, Phys. Rev. D 86, 074013 (2012) arXiv:1207.7052 [hep-ph]].

[5] T. Gutsche, M. A. Ivanov, J. G. Körner, V. E. Lyubovitskij and P. Santorelli, Phys. Rev. D 87, 074031 (2013) arXiv:1301.3737 [hep-ph]].

[6] T. Gutsche, M. A. Ivanov, J. G. Körner, V. E. Lyubovitskij and P. Santorelli, Phys. Rev. D 88, 114018 (2013) arXiv:1309.7879 [hep-ph]].

[7] T. Gutsche, M. A. Ivanov, J. G. Körner, V. E. Lyubovitskij and P. Santorelli, Phys. Rev. D 90, 114033 (2014)
arXiv:1410.6043 [hep-ph]].

[8] T. Gutsche, M. A. Ivanov, J. G. Körner, V. E. Lyubovitskij, P. Santorelli and N. Habyl, Phys. Rev. D 91, 074001 (2015) [Phys. Rev. D 91, 119907 (2015)] arXiv:1502.04864 [hep-ph]]; N. Habyl, T. Gutsche, M. A. Ivanov, J. G. Körner, V. E. Lyubovitskij and P. Santorelli, arXiv:1509.07688 [hep-ph].

[9] H. -Y. Cheng and B. Tseng, Phys. Rev. D 53, 1457 (1996) [Erratum-ibid. D 55, 1697 (1997)] hep-ph/9502391.

[10] H. -Y. Cheng, Phys. Rev. D 56, 2799 (1997) hep-ph/9612223.

[11] Fayyazuddin and Riazuddin, Phys. Rev. D 58, 014016 (1998) hep-ph/9802326.

[12] R. Mohanta, A. K. Giri, M. P. Khanna, M. Ishida, S. Ishida and M. Oda, Prog. Theor. Phys. 101, 959 (1999) hep-ph/9904324.

[13] C. -H. Chou, H. -H. Shih, S. -C. Lee and H. -n. Li, Phys. Rev. D 65, 074030 (2002) hep-ph/0112145.

[14] Z. -T. Wei, H. -W. Ke and X. -Q. Li, Phys. Rev. D 80, 094016 (2009) arXiv:0909.0100 [hep-ph]].

[15] L. Mott and W. Roberts, Int. J. Mod. Phys. A 27, 1250016 (2012) arXiv:1108.6129 [nucl-th]].

[16] F. Hussain, J. G. Körner, M. Krämer and G. Thompson, Z. Phys. C 51 (1991) 321.

[17] T. Mannel, W. Roberts and Z. Ryzak, Nucl. Phys. B 355 (1991) 38.

[18] F. Hussain, D. S. Liu, M. Krämer, J. G. Körner and S. Tawfiq, Nucl. Phys. B 370 (1992) 259.

[19] K. A. Olive et al. (Particle Data Group Collaboration), Chin. Phys. C 38, 090001 (2014).

[20] M. A. Ivanov, J. G. Körner, S. G. Kovalenko, P. Santorelli and G. G. Saidullaeva, Phys. Rev. D 85 (2012) 034004 arXiv:1112.3536 [hep-ph]].

[21] B. Aubert et al. (BaBar Collaboration), Phys. Rev. Lett. 96, 052002 (2006) hep-ex/0510070. 